Project Report

\title{
Development of a Pediatric Integrative Oncology Program: Pearls, Barriers, and Future Directions
}

Allison Ast ${ }^{1}$, Margaret Meyer ${ }^{2,}{ }^{+}$, R. Elyse Heidelberg ${ }^{3,+}{ }^{+}$, Jennifer M. Allen ${ }^{3,+}$, Amy Ly ${ }^{4,}{ }^{\dagger}$, Holly Spraker-Perlman ${ }^{5}{ }^{*}$

1. Hospitalist Medicine Program, St. Jude Children's Research Hospital, Memphis, TN, 38105, USA; E-Mail: Allison.Ast@STJUDE.ORG

2. Rehabilitation Services, St. Jude Children's Research Hospital, Memphis, TN, 38105, USA; E-Mail: Maggi.Meyer@STJUDE.ORG

3. Department of Psychology, St. Jude Children's Research Hospital, Memphis, TN, 38105, USA; EMails: Elyse.Heidelberg@STJUDE.ORG; Jennifer.Allen@STJUDE.ORG

4. Department of Pediatric Medicine, Division of Neurology, St. Jude Children's Research Hospital, Memphis, TN, 38105, USA; E-Mail: Amy.Ly@STJUDE.ORG

5. Department of Oncology, Division of Quality of Life and Palliative Care, St. Jude Children's Research Hospital Memphis, TN, 38105, USA; E-Mail: $\underline{\text { Holly.Spraker-Perlman@STJUDE.ORG }}$

+ These authors contributed equally to this work.

* Correspondence: Holly Spraker-Perlman; E-Mail: $\underline{\text { Holly.Spraker-Perlman@STJUDE.ORG }}$

Academic Editor: Leila Kozak

Special Issue: Integrative Therapies in Palliative Care

OBM Integrative and Complementary Medicine 2021, volume 6, issue 1

doi:10.21926/obm.icm.2101005
Received: December 02, 2020

Accepted: February 02, 2021

Published: February 08, 2021

\begin{abstract}
Children with cancer can experience intense suffering. Currently, despite our best efforts, symptoms associated with both cancer and its treatment result in physical, psychological, and emotional discomfort that can be difficult to manage with traditional Western medicine alone. Developing integrative services within a pediatric hematology-oncology program may help improve cancer-related symptoms by combining different modalities targeting overall health
\end{abstract}

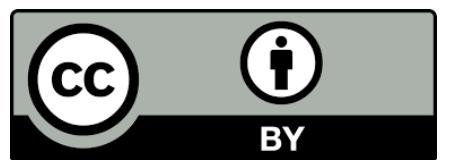

(C) 2021 by the author. This is an open access article distributed under the conditions of the Creative Commons by Attribution License, which permits unrestricted use, distribution, and reproduction in any medium or format, provided the original work is correctly cited. 
with traditional oncology care. This study details the initial development of a Pediatric Integrative Oncology (PIO) program at St. Jude Children's Research Hospital, which treats cancer and other catastrophic pediatric diseases. We highlight facilitators and barriers to integration of PIO. In addition, we advocate that PIO clinicians add to the growing literature base by sharing clinical and administrative experiences across institutions to support the longterm goal of developing specific guidelines that focus on using integrative modalities in children with cancer.

\section{Keywords}

Integrative; oncology; mindfulness; acupoint; acupuncture; acupressure; massage; yoga; pediatric

\section{Introduction}

The diagnosis of cancer in a child can be overwhelming and burdensome for families. Children suffer psychologically and physically during treatment, and at times symptoms are refractory to standard therapies. Currently, even the best traditional Western medicine systems cannot holistically provide physical, psychological, spiritual, and emotional care that affected families require. Therefore, as pediatric hematology-oncology, palliative care, and integrative medicine (IM) providers, we advocate for the early incorporation of integrative, low-risk, and evidence-informed modalities into traditional care, which can help families better cope with the challenges of their child's cancer diagnosis and its treatment. Integrative modalities may bring comfort, provide mental peace, alleviate physical symptoms, and strengthen the physician-family relationship in children with cancer. Adopting a robust IM program for pediatric hematology-oncology patients may yield meaningful opportunities to improve quality of life ( $Q \mathrm{OL})$ and address the gaps in care that families encounter.

Integrative oncology $(\mathrm{IO})$ is a burgeoning field that has been defined as "a patient-centered, evidence-informed field of cancer care that utilizes mind-body practices, natural products, and/or lifestyle modifications from different health traditions alongside conventional cancer treatments" [1]. The goal of $I O$ is to seamlessly pair conventional and complementary therapies during cancer treatment through survivorship to best serve patients and families in physical, psychological, emotional, spiritual, and social domains [2]. We believe that a program that provides tailored 10 services can synergistically help with symptom relief, impart control to patients and families, and support optimal health behaviors across the continuum of cancer care, from diagnosis through survivorship or during refractory cancer therapy and end-of-life care. To date, few studies have reported best practices for implementing 10 programs in a pediatric oncology (PO) setting in the United States (US). The objective of this report is to detail the initial development of a PIO program at an academic PO hospital and to determine the facilitators and barriers to its establishment.

\section{Materials and Methods/Results}


In this project conducted at St. Jude Children's Research Hospital (St. Jude), we developed a PIO consultation service and outpatient clinic in January 2021 to better address family's integrative questions by directing them to medical providers with specialty training in IM. We have two dualtrained physicians (fellowship training in both Pediatric Hematology-Oncology and Integrative Medicine) who collaborate with primary oncology teams at St. Jude to bridge the gap between conventional pediatric cancer care and IM modalities. To establish and offer beneficial integrative care, initial efforts have focused on developing modalities that were not historically offered at St. Jude, have evidence of benefit in pediatric cancer patients, and have been feasible to implement in other settings. St. Jude has a robust Child Life Services program including both pet-assisted therapy and music therapy programs prior to the official effort to establish a streamlined PIO service. Both of these modalities remain vital to holistic patient care and are included in our integrative offerings, but will not be discussed in this paper.

The five main integrative service domains under development at our institution are a PIO consultation team, yoga instruction, massage therapy, acupoint services, and mindfulness training. As minimizing the risks to children during therapy is paramount, some integrative practices used in adults with cancer and/or healthy children may not be appropriate in the care of severely immunocompromised pediatric patients. Until there are reliable data on the appropriate dosing, benefits, and safety of herbal medications/supplements for children with cancer, our PIO team discusses the use of natural products with patients and families, but we are not routinely recommending supplements or herbal medications for immunocompromised children receiving chemotherapy. However, PIO consultation allows the opportunity for parental disclosure of supplement use, and bi-directional information exchange on safety and potential unmet needs in a non-judgmental environment.

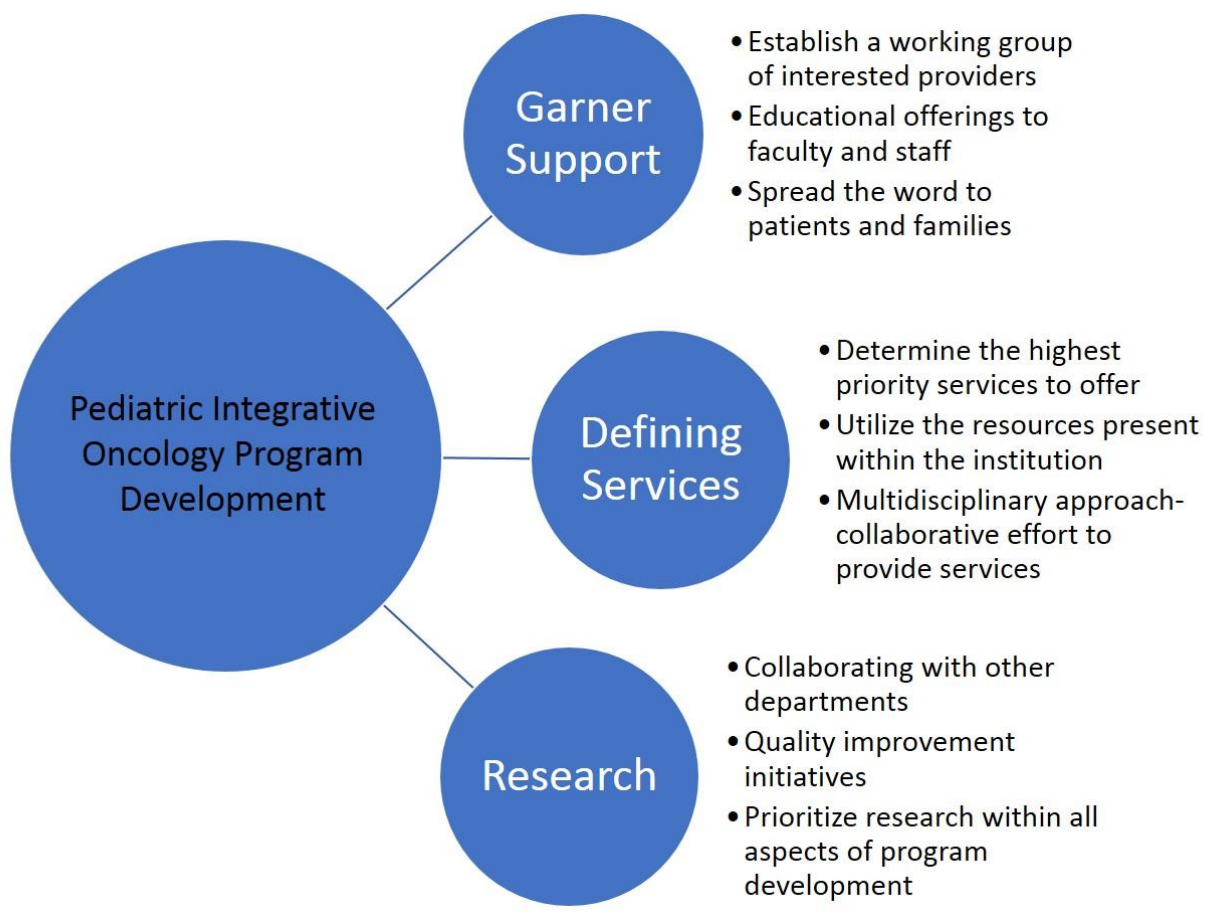

Figure 1 Program Development Pearls

\section{Garner Support}




\subsection{Seek Administrative Backing, Multi-Disciplinary Healthcare Provider Interest, and Patient/Family Support}

An important first step in developing a PIO clinical program is establishing a hospital-wide, multidisciplinary working group. The administration at St. Jude was open to creating this provider group, and multi-disciplinary team members were given time to attend meetings and achieve individual or unit goals by participating in various projects. Our Integrative Medicine Working Group (IMWG) began meeting monthly in January 2018 and transitioned to a Web-based platform after onset of the COVID-19 pandemic. The IMWG comprises parent representatives and diverse healthcare professionals, including psychologists, occupational, physical, and music therapists, child life specialists, social workers, chaplains, dieticians, nurses, nurse practitioners, and physicians. An open invitation to interested staff allowed creation of a group of like-minded individuals with diverse skill sets that may have been previously underutilized. Members of the IMWG have championed the creation and implementation of current programs in development for PIO services at St. Jude. In our opinion, by engaging interested staff and highlighting their talents and accomplishments, we have built new integrative services for patients using existing resources. The need for additional personnel and support for a robust IM program cannot be understated, yet piloting PIO services with a dedicated small group of providers has allowed the collection of objective data detailing patient needs, utilization, and satisfaction with PIO services, which is expected to propel the program forward.

\subsection{Listen to Your Colleagues and Your Patients/Families}

The IMWG designed and implemented needs assessments for both healthcare providers (HCPs) and patients/families at St. Jude. Our data on HCPs was collected through an institutionally sponsored survey (via DataSTAT) to determine the services that would be championed by referring clinicians and those that were concerning for HCPs. In general, pediatric HCPs were interested in integrating services that they deemed to be evidence-based and have a low risk of complications in very ill PO patients (manuscript in progress). In particular, massage therapy and yoga were considered safe and needed in our patient population.

We also engaged with patients and families. St. Jude has an active Patient \& Family Advisory Committee (PFAC), a Bereaved Parent Steering Council (BPSC), and a patient app (Our St. Jude) that allows clinicians to ask for family feedback in real time. The IMWG engaged the PFAC early and presented ideas several times to solicit input on programming. The BPSC also discussed ideal services for patients with no further cancer-directed therapy options. Finally, we conducted a survey using our patient app to inquire about needs and determine the services of interest from all current St. Jude families. Families $(n=56)$ were overwhelmingly supportive of program development, and preferred (in descending order) support for dietary and herbal counselling, massage therapy, yoga, acupressure/acupuncture, mindfulness, and aromatherapy (internal data). Of note, $60 \%(\mathrm{~N}=33$ ) of parents are currently using (or have used) integrative modalities for their children during cancer therapy; of parents who responded positively, $76 \%(\mathrm{~N}=42)$ found integrative services independent of their cancer providers (internal data). We are currently planning a mixed-method study to more rigorously identify patient/family needs and beliefs about PIO services to further guide programming. 


\subsection{Spread the Word to Patients, Families, Referring Providers, and Staff at Your Institution}

The St. Jude PIO program is currently embedded in and modelled after our Pediatric Palliative Care (PPC) program [3]. The PPC team has been successful in influencing institutional culture and offering services to patients with the greatest need by providing education, creating policies and guidelines, targeting services for specific populations, and providing consultations.

Our broadest base goal is to maximize numbers through educating patients, families, and healthcare faculty and staff about integrative medicine. The IMWG has open educational offerings every two to three months, participates in all-invited institutional educational platforms, and recently sponsored a Grand Rounds lecture. Spreading interest in this program, providing evidence of safety and efficacy of utilized modalities, and working with referring colleagues have been vital strategies to incorporate PIO into our institutional culture. Our overarching goal was to create a comprehensive clinical consultation service with supportive clinical programs in PIO domains for patient use. Considering that the number of consults performed is dictated by staff numbers, time/effort spent, and funding, we aim to train and use IMWG clinicians in the domain programs defined below to ensure a maximum effect. In the future, we hope to recruit additional HCPs (e.g., licensed massage therapists), but currently both funding and the COVID-19 pandemic have precluded this.

\section{Define Services to Provide}

As above, we have focused initial efforts on four domain areas for patient services after providing educational offerings for interested staff members. These services represented previously unsponsored interventions that overlapped in their clinician support in terms of safety, evidence, and highest patient/family need.

Using available literature, parent and family input, and clinician guidance, we defined the initial services priorities to include yoga, massage, acupoint services, and mindfulness training, which are discussed below in detail.

\subsection{Yoga}

Children with cancer are at high risk for loss of mobility, fitness, balance, and strength due to decreased physical activity as they undergo treatment [4,5]. Common side effects of cancer and its treatment which may compound patients' functional decline include pain, depression, anxiety, weakness, and fatigue. Several studies provide evidence that yoga may offer psychosocial benefits for pediatric cancer patients [6-8], in addition to improving cancer-related fatigue [9], physical fitness, and activity levels [10].

At St. Jude, the use of yoga as a mind-body intervention is led by two Doctors of Physical Therapy (DPT), one who was previously trained as a yoga instructor (Registered Yoga Teacher, RYT-200) and had additional training through the Young Warriors Children's Yoga Teachers Training collaborative. Both DPTs completed 60 hours in the Yoga Calm Youth Instructor Certification Program and are now using yogic breath work and asanas (postures) in their standard physical therapy sessions with interested patients.

The goals of the yoga program are 1) to incorporate yoga into physical therapy services as oneon-one sessions for interested and eligible pediatric cancer patients; 2) to offer group classes for St. 
Jude patients and families in both inpatient and outpatient settings; and 3) to develop a Web-based yoga curriculum for home use that targets common physical complaints seen in-cancer patients and survivors. The creation of the yoga program was supported by internal grant funding to include materials for group programming (e.g., mats, straps, blocks, bolsters) and provider training. A consent form for participation, guidelines for participant eligibility, and a quality improvement (QI) project assessing the uptake of the home-based yoga curriculum and patient/family satisfaction with yoga offerings have been developed. Although the current COVID-19 pandemic has slowed the implementation of yoga group classes, one-on-one yoga sessions are available and Web-based video content has been created.

\subsection{Massage}

Many studies suggest that massage therapy may help modify symptoms experienced by patients with cancer. However, there is limited rigorous research on establishing the true benefits of massage therapy. In a review of 24 randomized controlled trials (RCTs) of massage in children with cancer, more than half did not report data sufficient for calculation of effect size [11]. However, data suggest that massage therapy decreases anxiety (immediately after the massage), and this effect is additive in subsequent massage sessions [11]. Similarly, studies show statistically significant reductions in heart rate [12], anxiety [12,13], and days to engraftment in pediatric bone marrow transplantation [13] when massage is incorporated into oncology care. Finally, despite the heterogeneity in study design to date, systematic reviews show that massage in children with cancer can decrease pain, nausea, stress, and anxiety [14].

The goals of our massage programming are to 1 ) provide massage services for patients with musculoskeletal pain, insomnia, stress/anxiety, and/or the desire for massage, and 2) document the feasibility of using bedside clinicians as parent massage educators. At St. Jude, interested bedside HCPs were trained in pediatric massage techniques for the purpose of teaching caregivers simple techniques during routine clinical care. To date, approximately 50 providers across 12 disciplines have completed the Certified Pediatric Massage Therapist Training program (CPMT-1) through the Liddle Kidz Foundation. In addition, two QI projects are underway to assess the feasibility and acceptability of integrating nurse-provided hand or foot massage for admitted patients and patients undergoing sedated procedures in outpatient procedure rooms. Changes in staffing during the COVID-19 pandemic and integration of massage into current nursing practice have posed challenges in implementing bedside massage St. Jude. Future plans include credentialing and incorporating licensed massage therapists for more formal massage services programs. In addition, our team plans to partner with local massage therapy schools to precept students' at St. Jude to allow for parent/caregiver massage services as well.

\subsection{Acupoint Services}

Acupoint services comprise acupuncture (needle- or laser-based) or acupressure (manual pressure) to stimulate classically defined acupoints for wellness and/or symptom management. Acupoint is one domain of traditional Chinese medicine and is utilized frequently in the US. In 2015, over three million patients visited acupuncturists for various health-related conditions [15]. The National Institutes of Health (NIH) Consensus Conference on Acupuncture supports the safety and efficacy of acupuncture in treating chemotherapy-induced nausea and vomiting (CINV) [14]. Several 
clinical trials in adults with cancer have found acupuncture to be effective for managing nausea, vomiting, post-operative pain, and headache [16-20]. Acupuncture is now a recommended modality for complementary use in the NCCN guidelines for antiemesis, cancer pain in adults, and cancerrelated fatigue [21-23]. Finally, the risk of serious adverse events (AEs) is very low [24, 25].

Few high-quality studies support the benefits of acupuncture in children with cancer, but several have shown effectiveness in CINV [25]. Other retrospective data have documented the safety and feasibility of acupuncture and acupressure in the care of PO patients [26-28]. Barriers to using acupuncture include risk of bleeding and/or infection, limitations in data to guide appropriate blood count parameters, and patient/family and clinician concerns about the use of needles [26]. Studies show that PO patients receiving acupuncture, even during times of severe thrombocytopenia, have minimal evidence of bleeding [27]. In addition, no increases in severe AEs (including infection) have been documented for $\mathrm{PO}$ patients receiving acupuncture compared to similar patients receiving standard care at several academic pediatric institutions [27, 28]. Finally, patients can safely receive acupressure or laser acupuncture treatment without the use of needles regardless of blood count parameters. Overall, we advocate for adding acupoint services as a complementary modality and for conducting further research on its benefits and safety in immunocompromised children.

We are developing an evidence-informed acupoint program offering both acupressure and acupuncture based on patient clinical status, and patient, caregiver, and physician preferences. The primary goal of this program is to provide an additional evidence-informed modality for common refractory side effects seen during and after treatment. Initially, we established a relationship with a community-based physician acupuncturist. By educating the administration and staff, we were able to credential this medical acupuncturist to perform services at our institution. In addition, one of our PIO physicians completed the coursework necessary for physician acupuncturists. We have developed guidelines for acupoint use, informed consent documents, acupuncture-specific medical record documentation, and educational materials for patients and families. We are currently providing acupoint services in both inpatient and outpatient spaces, although only during specific blocks of time due to availability. We have designed a QI mechanism to collect outcomes for patients opting for this new service and hope to share this data, when complete.

\subsection{Mindfulness}

Pediatric cancer patients experience a breadth of physical and psychological symptoms that often persist into survivorship [29-31]. Despite this, the psychosocial needs of these patients, especially adolescent and young adults, are often unmet [32,33]. Mindfulness refers to a state of mind that involves compassionate, non-judgmental, and present-focused awareness of one's experiences, and has the potential to address the physical and psychological symptoms experienced by pediatric cancer patients and survivors of childhood cancer. Mindfulness-based interventions guide patients to increase non-judgmental awareness of thoughts, feelings, and sensations, and strengthens their capacity to respond in a more accepting, conscious, and skillful manner [34]. In adult cancer patients, mindfulness has been found to reduce stress, anxiety and depression, and improve mood, sleep, fatigue and well-being [35-37]. Less research has focused on mindfulness for pediatric cancer patients, though results on the benefits of mindfulness for youth are promising [38]. For PO patients, evidence suggests that mindfulness training reduces emotional distress, improves QoL, and reduces negative self-perception [39]. 
St. Jude has an extensive team of psychologists that helps patients and families cope with various aspects of pediatric cancer diagnosis and treatment. Psychologists offer a variety of interventions for anxiety, mood concerns, and behavioral problems. They also offer non-pharmacologic strategies to augment physical and psychological symptoms, including mindfulness, relaxation techniques, biofeedback, guided imagery, clinical hypnosis, and other standard evidence-based interventions, such as cognitive behavioral therapy.

To enhance our capabilities to teach mindfulness to patients, families, and faculty/staff, two of our HCPs are completing training in a mindfulness-based intervention (Stress Management and Resiliency Training) through the Benson-Henry Institute for Mind-Body Medicine at Massachusetts General Hospital. Our goal is to offer structured mindfulness groups for both patients and caregivers to bolster their coping skills as they navigate a cancer diagnosis and treatment. A secondary goal is to offer mindfulness programming for clinicians with hope that this may bolster resiliency in healthcare professionals at St. Jude.

\section{Research}

For all the above domains, QI data are being collected to ensure that clinical programs lead to a meaningful change in patients, families, and staff. From a provider perspective, documenting the experience of sharing integrative skills with patients is imperative to inform clinicians across the globe. In our experience, adding non-pharmacologic tools to a HCP's skill set has been welcomed and anecdotally helpful for job satisfaction rather than an additional burden. Two goals in each QI project are to ensure that workflow in a clinic or unit is not negatively affected by implementation of an integrative modality, and evaluating patient, family, and staff satisfaction with the new care provided. We strongly recommend collecting and publishing all evidence to document both benefits and any adverse events (AE) associated with integrative modalities to help guide the development of PIO programs nationally.

\section{Discussion}

An increasing number of hospitals are offering complementary or integrative health services. However, clear guidance about how to successfully and efficiently integrate these services into conventional healthcare settings is not established. Previous work has identified challenges associated with designing, initiating, and maintaining IM programs, such as clinical and administrative barriers, inertia or a general resistance to change, lack of familiarity with nontraditional HCPs, historical enmity between physicians and other provider groups, skepticism about treatments, lack of funding, perceived or actual lack of provider regulation, and heterogeneity of products and services [40]. Developing a PIO program in an established hospital setting comes with many challenges and requires dedicated work. We have met the following barriers during our program development:

\subsection{Lack of Definition of Integrative vs Complementary vs Alternative}

One barrier to developing a PIO program is the lack of a concise definition of PIO, what modalities it may support, and its role in conventional pediatric cancer care. The Society for Integrative Oncology (SIO) pediatric sub-committee has been crafting a cohesive definition for PIO, but 
currently a consensus definition or guidelines specific to PIO are lacking. Integration of IM into PO, even as an adjunct for symptom management, is controversial due to the rigorous evidence used to guide cancer treatments and concerns about introduction of complicating factors into the care of immunocompromised children. In addition, as families become more involved in searching for alternative cancer therapies, physicians may be skeptical about the care certain IM providers may offer to their patients. Our program offers low-risk complementary services alongside traditional care and NOT alternative therapies, those used in lieu of traditional cancer, or those that have no evidence of safety. Our team has clearly communicated with referring providers about what we do and do not offer to work collaboratively with attending oncologists [41].

As mentioned above, a PIO program can develop without recommendations of oral botanicals or supplements. Our program focuses on non-pharmacological therapies that are generally considered safe and have little to no risk of interfering with conventional therapies. Evidence supporting the safety and efficacy of herbal and biological treatments alongside chemotherapy in children is weak. The manufacturing of herbal products is not regulated as strictly as for pharmaceuticals, and systematic reporting of AEs by users is not legally mandated [42]. Finally, some side effects of and interactions between herbal medications and chemotherapy have been documented in adult cancer patients, yet there are not enough pediatric specific data to support routine use during cancer therapy with curative intent [43]. We allow for exceptions to this rule on an individual basis and in conjunction with primary oncologists, based on the disease trajectory of patients. Significantly more data are needed to move herbal products into frontline therapy in $\mathrm{PO}$, and in particular for patients on therapeutic cancer trials.

\subsection{Lack of Rigorous Research in PIO Programs}

Theoretical approaches to health and well-being are conceptualized differently in IM than in Western medicine, and thus RCTs of integrative therapies can be challenging to design and implement $[44,45]$. Conducting research on the efficacy of complementary therapies requires unique considerations such as underlying medical theories, heterogeneity of products and practices, individualized therapy based on patient's presentation and symptoms, and implementation of appropriate placebo controls. Significant opportunities remain to expand the evidence base for integrating complementary therapies into the care of children with cancer. We believe that well designed, multi-institutional trials will be needed to provide the highest quality evidence for many PIO services.

\subsection{Lack of Support from Oncologists for PIO Programs}

Another factor hindering the development of PIO programs is oncologist's lack of awareness and education about the importance of PIO. For busy oncologists, staying abreast of new complementary research and the ever-expanding world of complementary therapies to cancer treatment is a challenge. Despite the increasing demand for and interest in integrative care, systematic education for HCP in complementary and integrative therapies for pediatric patients is still lacking [46]. Understanding provider beliefs concerning IM and developing targeted educational initiatives can lead to improved collaboration. Building a cooperative relationship with referring providers and being transparent about recommendations for patients is one way by which we have established a referral base [41]. 


\subsection{Financial Coverage for Integrative Services}

A significant barrier to program maintenance is the financial sustainability of IM services. At St. Jude, we are fortunate to have funding through generous donors and internal grants, which cover the cost of care for families. A review of IO programs found that most programs provided some IM therapies free of charge to patients, by using various funding strategies such as foundation and grant support. Other sites use hospital budgetary funds, third-party billing, and direct billing to patients to cover $[47,48]$. At the national level, the field of IM made prominent strides with the Patient Protection and Affordable Care Act (HR 3590), which was slated to provide opportunities for integrative healthcare growth, particularly in terms of coverage for health promotion and public health strategies. Yet this legislation is in jeopardy, and it is unclear how our healthcare system will respond to future legislation. We encourage programs to start with feasible goals, utilize the skills of their current workforce, and work to prove utility, as this has helped power our IM services. We believe that high-quality data showing efficacy and utility of IM services may eventually lead to insurance coverage of certain modalities. However, without a broad-based change in healthcare policy at the national level, funding will remain the largest barrier to implementing services in traditional healthcare settings.

\section{Conclusions}

When a child is ill, especially with a devastating disease like cancer, many parents want to be proactive in their child's care and consider IM modalities. In parallel with and as a response to that trend, the field of PIO has emerged to ensure that patients have access to evidence-based, safe, comprehensive, and patient-centered care throughout the disease trajectory. A PIO team may provide information and support to patients and caregivers and remove the burden from primary oncology teams to educate families about interventions considered less mainstream.

To meet patient and family needs, we developing a formal PIO program at St. Jude. Forming the IMWG was a vital first step that allowed a multi-disciplinary team of providers to collect data from caregivers and healthcare colleagues on incorporating integrative modalities in cancer care. This team has implemented complementary services that were not previously available and continues to strive for holistic care for our patients. Through our consultation service, we have created a nonjudgmental space for education and dialogue about alternative therapies that may be suggested by well-meaning family members or discovered using non-scientific internet searches. There are currently no published guidelines for PIO program development or implementation. However, we have outlined herein the progress made to implement four evidence-based, low-risk modalities at our institution. Our program is continuously refined, and as with any new clinical service, there have been challenges and setbacks. There have also been opportunities for collaboration and innovation providing major victories for patients, families, and clinicians.

PIO is a becoming a subspecialty within the pediatric IM subspecialty that requires further tailored research and educational efforts specific to caring for children with compromised immune systems. Pediatric academic institutions have the opportunity to develop, integrate, and study initiatives to improve symptomatic care, meet family's information needs, and improve health outcomes through integrative healthcare. Collaboration across institutions is critical to the success of PIO in hospital settings. Finally, PIO clinicians have the responsibility to collect and publish their 
experience with PIO clinical care and program development to help shape programs and answer questions about the optimal use and delivery of integrative modalities to this highly specialized population.

\section{Author Contributions}

Dr. Allison Ast - conceptualizing project, literature review, data collection and analysis, primary manuscript drafting, primary manuscript editing and publication tasks.Dr. Margaret Meyer conceptualizing project, literature review, primary manuscript drafting, editing and publication tasks. Elyse Heidelberg - conceptualizing project, literature review, primary manuscript drafting, primary manuscript editing. Dr. Jennifer Allen - conceptualizing project, literature review, primary manuscript drafting, and manuscript editing. Dr. Amy Ly - conceptualizing project, literature review, primary manuscript drafting, primary manuscript editing. Dr. Holly Spraker-Perlman conceptualizing project, literature review, data collection and analysis, primary manuscript drafting, primary manuscript editing and publication tasks, mentorship to first author.

\section{Competing Interests}

The authors have declared that no competing interests exist.

\section{References}

1. Witt CM, Balneaves LG, Cardoso MJ, Cohen L, Greenlee H, Johnstone $P$, et al. A comprehensive definition for integrative oncology. J Natl Cancer Inst Monogr. 2017; 2017: Igx012.

2. National Center for Complementary and Integrative Health (NCCIH) [Internet]. 2020. Available fromhttps://nccih.nih.gov

3. Kaye EC, Friebert S, Baker JN. Early integration of palliative care for children with high-risk cancer and their families. Pediatr Blood Cancer 2016; 63: 593-597.

4. Winter C, Müller C, Hoffmann C, Boos J, Rosenbaum D. Physical activity and childhood cancer. Pediatr Blood Cancer. 2010; 54: 501-510.

5. Wright MJ, Galea V, Barr RD. Proficiency of balance in children and youth who have had acute lymphoblastic leukemia. Phys Ther. 2005; 85: 782-790.

6. Geyer R, Lyons A, Amazeen L, Alishio L, Cooks L. Feasibility study: The effect of therapeutic yoga on quality of life in children hospitalized with cancer. Pediatr Phys Ther. 2011; 23: 375-379.

7. Moody K, Daswani D, Abrahams B, Santizo R. Yoga for pain and anxiety in pediatric hematologyoncology patients: Case series and review of the literature. J Soc Integr Oncol. 2010; 8: 95-105.

8. Thygeson MV, Hooke MC, Clapsaddle J, Robbins A, Moquist K. Peaceful play yoga: Serenity and balance for children with cancer and their parents. J Pediatr Oncol Nurs. 2010; 27: 276-284.

9. Hilfiker R, Meichtry A, Eicher M, Balfe LN, Knols RH, Verra ML, et al. Exercise and other nonpharmaceutical interventions for cancer-related fatigue in patients during or after cancer treatment: A systematic review incorporating an indirect comparisons meta analysis. Br J Sports Med. 2018; 52: 651-658.

10. Wurz A, Chamorro-Vina C, Guilcher G, Schulte F, Culos-Reed SN. The feasibility and benefits of a 12-week yoga intervention for pediatric cancer out-patients. Pediatr Blood Cancer. 2014; 61: 1828-1834. 
11. Beider S, Moyer CA. Randomized controlled trials of pediatric massage: A review. Evid Based Complement Altern Med. 2007; 4: 23-34.

12. Post-White J, Fitzgerald M, Savik K, Hooke MC, Hannahan AB, Sencer SF. Massage therapy for children with cancer. J Pediatr Oncol Nurs. 2009; 26: 16-28.

13. Rodriguez-Mansilla J, Gonzalez-Sanchez B, Torres-Piles S, Martín JG, Jiménez-Palomares M, Bellino MN. Effects of the application of therapeutic massage in children with cancer: $A$ systematic review. Rev Lat Am Enfermagem. 2017; 25: e2903

14. Bowman MA. Acupuncture. JAMA. 1998; 280: 1518-1524.

15. Black LI, Clarke TC, Barnes PM, Stussman BJ, Nahin RL. Use of complementary health approaches among children aged 4-17 years in the United States: National Health Interview Survey, 2007-2012. Natl Health Stat Report. 2015; 78: 1-19.

16. Garcia MK, McQuade J, Haddad R, Patel S, Lee R, Yang P, et al. Systematic review of acupuncture in cancer care: A synthesis of the evidence. J Clin Oncol. 2013; 31: 952-960.

17. World Health Organization. Acupuncture: Review and analysis of reports on controlled clinical trials. Geneva: World Health Organization; 2002.

18. Ezzo JM, Richardson MA, Vickers A, Allen C, Dibble S, Issell BF, et al. Acupuncture-point stimulation for chemotherapy-induced nausea or vomiting. Cochrane Database Syst Rev. 2006: CD002285.

19. Dean-Clower E, Doherty-Gilman AM, Keshaviah A, Baker F, Kaw C, Lu W, et al. Acupuncture as palliative therapy for physical symptoms and quality of life for advanced cancer patients. Integr Cancer Ther. 2010; 9: 158-167.

20. Cohen AJ, Menter A, Hale L. Acupuncture: Role in comprehensive cancer care-a primer for the oncologist and review of the literature. Integrat Cancer Ther. 2005; 4: 131-143.

21. Antiemesis [Internet]. National Comprehensive Cancer Network; 2020. Available from: https://www.nccn.org/professionals/physician gls/pdf/antiemesis.pdf

22. Adult Cancer Pain [Internet]. National Comprehensive Cancer Network; 2020. Available from: https://www.nccn.org/professionals/physician gls/pdf/pain.pdf

23. Cancer-Related Fatigue [Internet]. National Comprehensive Cancer Network; 2020. Available from: https://www.nccn.org/professionals/physician gls/pdf/fatigue.pdf

24. Cybularz PA, Brothers K, Singh GM, Feingold JL, Lewis ME, Niesley ML. The safety of acupuncture in patients with cancer therapy-related thrombocytopenia. Med Acupunct. 2015; 27: 224-229.

25. Witt CM, Pach D, Brinkhaus B, Wruck K, Tag B, Mank S, et al. Safety of acupuncture: Results of a prospective observational study with 229,230 patients and introduction of a medical information and consent form. Forsch Komplementmed. 2009; 16: 91-97.

26. Jindal V, Ge A, Mansky PJ. Safety and efficacy of acupuncture in children: A review of the evidence. J Pediatr Hematol Oncol. 2008; 30: 431-442.

27. Ladas EJ, Rooney D, Taromina K, Ndao DH, Kelly KM. The safety of acupuncture in children and adolescents with cancer therapy-related thrombocytopenia. Support Care Cancer. 2010; 18: 1487-1490.

28. Chokshi SK, Ladas EJ, Taromina K, McDaniel D, Rooney D, Jin Z, et al. Predictors of acupuncture use among children and adolescents with cancer. Pediatr Blood Cancer. 2017; 64: e26424.

29. Brinkman TM, Zhu L, Zeltzer LK, Recklitis CJ, Kimberg C, Zhang N, et al. Longitudinal patterns of psychological distress in adult survivors of childhood cancer. Br J Cancer. 2013; 109: 1373-1381. 
30. Huang IC, Brinkman TM, Kenzik K, Gurney JG, Ness KK, Lanctot J, et al. Association between the prevalence of symptoms and health-related quality of life in adult survivors of childhood cancer: A report from the St Jude Lifetime Cohort study. J Clin Oncol. 2013; 31: 4242-4251.

31. Ljungman G, Gordh T, Sorensen S, Kreuger A. Pain variations during cancer treatment in children: A descriptive study. Pediatr Hematol Oncol. 2000; 17: 211-221.

32. Zebrack BJ, Corbett V, Embry L, Aguilar C, Meeske KA, Hayes-Lattin B, et al. Psychological distress and unsatisfied need for psychosocial support in adolescent and young adult cancer patients during the first year following diagnosis. Psychooncology. 2014; 23: 1267-1275.

33. Van der Gucht K, Takan K, Labarque V, Vandenabeele K, Nolf N, Kuylen S, et al. A mindfulnessbased intervention for adolescents and young adults after cancer treatment: Effects on quality of life, emotional distress, and cognitive vulnerability. J Adolesc Young Adult Oncol. 2017; 307317

34. Kabat-Zinn J. Full catastrophe living: How to cope with stress, pain and illness using mindfulness meditation. London: Piatkus; 1990.

35. Wurtzen H, Dalton SO, Elsass $P$, Sumbundu AD, Steding-Jensen M, Karlsen RV, et al. Mindfulness significantly reduces self-reported levels of anxiety and depression: Results of a randomized controlled trial among 336 Danish women treated for stage I-III breast cancer. Eur J Cancer. 2013; 49: 1365-1373.

36. Nakamura Y, Lipschitz DL, Kuhn R, Kinney AY, Donaldson GW. Investigating efficacy of two brief mind-body intervention programs for managing sleep disturbance in cancer survivors: A pilot randomized trial. J Cancer Surviv. 2013; 7: 165-182.

37. Van der Lee ML, Garssen B. Mindfulness-based cognitive therapy reduces chronic cancerrelated fatigue: A treatment study. Psychooncology. 2012; 21: 264-272.

38. Klingbeil DA, Renshaw TL, Willenbrink JB, Copek RA, Chan KT, Haddock A, et al. Mindfulnessbased interventions with youth: A comprehensive meta-analysis of group-design studies. J Sch Psychol 2017; 63: 77-103

39. Van der Gucht K, Takano K, Labarque V, Vandenabeele K, Nolf N, Kuylen S, et al. A mindfulnessbased intervention for adolescents and young adults after cancer treatment: Effects on quality of life, emotional distress, and cognitive vulnerability. J Adolesc Young Adult Oncol. 2017; 6: 307-317.

40. Vohra S, Feldman K, Johnston B, Waters K, Boon H. Integrating complementary and alternative medicine into academic medical centers: Experience and perceptions of nine leading centers in North America. BMC Health Serv Res. 2005; 5: 78.

41. Ben-Ayre E, Schiff E, Shapiae C, Frenkelf M, Shalom T, Steiner M. Modeling an integrative oncology program within a community-centered oncology service in Israel. Patient Educ Couns. 2012; 89: 423-429.

42. Kelly KM. Integrative therapies for children with hematological malignancies. Hematology Am Soc Hematol Educ Program. 2009; 2009: 307-312.

43. Sencer SF, Kelly KM. Complementary and alternative therapies in pediatric oncology. Pediatr Clin North Am. 2007; 54: 1043-1060.

44. Melnick SJ. Developmental therapeutics: Review of biologically based complementary and alternative medicine (CAM) therapies for potential application in children with cancer-part II. J Pediatr Hematol Oncol. 2006; 28: 271-285. 
45. Melnick SJ. Developmental therapeutics: Review of biologically based CAM therapies for potential application in children with cancer: Part I. J Pediatr Hematol Oncol. 2006; 28: 221-230.

46. McClafferty H. Integrative pediatrics: Looking forward. Children (Basel). 2015; 2: 63-65.

47. Seely DM, Weeks LC, Young S. A systematic review of integrative oncology programs. Curr Oncol. 2012; 19: e436-e461.

48. Sagar SM. Integrative oncology in North America. J Soc Integr Oncol. 2006; 4: 27-39.

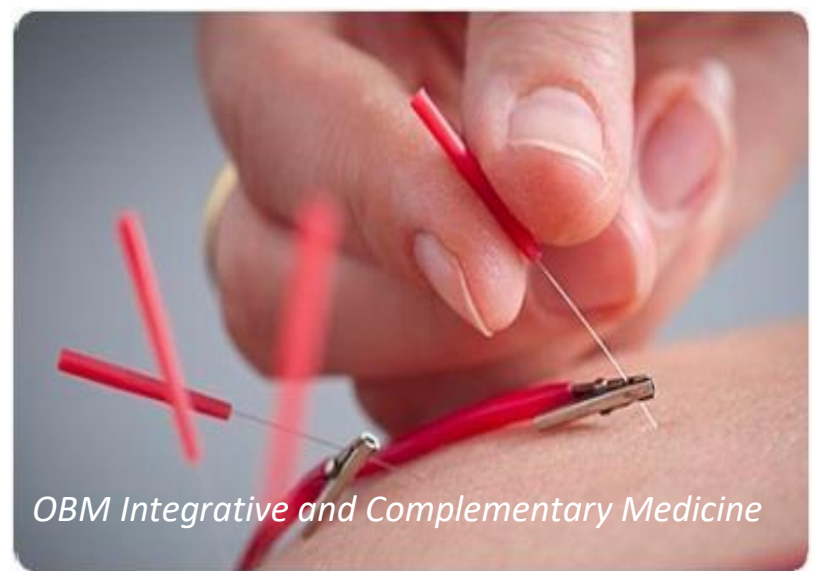

Enjoy OBM Integrative and Complementary Medicine by:

1. Submitting a manuscript

2. Joining in volunteer reviewer bank

3. Joining Editorial Board

4. Guest editing a special issue

For more details, please visit:

http://www.lidsen.com/journals/icm 